\title{
Exposure of Fluoride in Coal Basin
}

\author{
Khageshwar Singh Patel1", Ankit Yadav1, Keshaw Prakash Rajhans', Shobhana Ramteke1, \\ Reetu Sharma1, Irena Wysocka², Irena Jaron² \\ ${ }^{1}$ School of Studies in Chemistry/Environmental Science, Pt. Ravishankar Shukla University, Raipur, India \\ ${ }^{2}$ Polish Geological Institute, Rakowiecka, Warsaw, Poland \\ Email: patelks_55@hotmail.com
}

Received 12 November 2015; accepted 16 February 2016; published 19 February 2016

Copyright (C) 2016 by authors and Scientific Research Publishing Inc.

This work is licensed under the Creative Commons Attribution International License (CC BY). http://creativecommons.org/licenses/by/4.0/

c) (7) Open Access

\begin{abstract}
Coal is a dirty fuel contaminated with $\mathrm{F}^{-}$and other elements. Several million tons of coal are burnt in Korba basin, central India to generate electricity with pouring fluorine and other elements into the environment. The water is contaminated with $\mathrm{F}^{-}$and other chemicals beyond the permissible limits. The contaminated water is consumed by the human and animals by excreting the balanced toxicants through the stool and urine. Several folds higher concentration of $F^{-}$in urine $(44 \mathrm{mg} / \mathrm{L})$ and stool $(266 \mathrm{mg} / \mathrm{kg})$ samples of the cattle are observed. The prevalence of fluorosis diseases in cattle of the Korba basin is reported.
\end{abstract}

\section{Keywords}

\section{Coal, Fluoride, Contamination, Toxicity}

\section{Introduction}

Coal is a dirty fuel contaminated with toxic elements at the traces [1] [2]. The fluoride content in the coals is ranged from $20-500 \mathrm{mg} / \mathrm{kg}$ [3]. The environments in coal burning areas were severely contaminated with $\mathrm{F}^{-}$ and other toxicants [4]-[9]. The human and other animals living in the coal burning areas were affected by chronic endemic fluorosis diseases due to the excessive intake of $\mathrm{F}^{-}$from air, water and food [10]-[12]. Several states of the country are suffered with fluorosis diseases due to intake of contaminated groundwater [13]-[20]. In the present work, the contamination of ground and surface water of the largest coal basin of India with $\mathrm{F}^{-}$ and other elements is described. The exposure assessment of the $\mathrm{F}^{-}$toxicity in the domestic animals is described.

\footnotetext{
${ }^{*}$ Corresponding author.
}

How to cite this paper: Patel, K.S., Yadav, A., Rajhans, K.P., Ramteke, S., Sharma, R., Wysocka, I. and Jaron, I. (2016) Exposure of Fluoride in Coal Basin. International Journal of Clean Coal and Energy, 5, 1-12. 


\section{Materials and Methods}

\subsection{Study Area}

Korba basin, CG, India $\left(22^{\circ} 21^{\prime} 0^{\prime \prime} \mathrm{N}\right.$ and $\left.82^{\circ} 40^{\prime} 48^{\prime \prime} \mathrm{E}\right)$ has rich deposit of coal over $\approx 1.0 \times 10^{4} \mathrm{~km}^{2}$ area. It is a power capital of Chhattisgarh state, India. The basin is composed of four blocks: Kartala, Katghora, Korba and Pali with population of $\approx 1$ million distributed over 710 cities, towns and villages. A huge amount of coal > $10000 \mathrm{MT} / \mathrm{Yr}$ has been consumed by various units of thermal power plants for generation of $\approx 40000 \mathrm{KW}$ electricity. At least 12 open and underground coal mines are in operation with annual production of $\approx 3$ BT coal by discharging > $100 \mathrm{BT} / \mathrm{Yr}$ liquid effluents in the environment. In addition, the Asia biggest Aluminum Plant (BALCO) is running in the Korba basin.

\subsection{Sample Collection}

The main river flowing through Korba basin is the Hasdeo River. The Bango dam has been constructed across this river through which water flows into canals. Several pit lakes are found to exist in the Korba basin due to mining out of the coal. The ground and surface water samples were collected from 30 and 6 locations of the basin in month of January, 2013 [21] (Figure 1). A 1-L cleaned narrow mouth polyethylene bottle was used for the collection. The container was rinsed three times with the sample water and filled with the water up to the mouth.

The physical parameters i.e. temperature (T), pH, dissolved oxygen (DO), reduction potential (RP) and electrical conductivity (EC) of the water were measured at the spot by using HANNA sensors.

The first morning stool $(1 \mathrm{~kg})$ of the domestic animals $(\mathrm{n}=20)$ was collected manually. The stool sample was stored in the polyethylene bottle and dried in oven at $60^{\circ} \mathrm{C}$ till the dryness. They were crushed and sieved out the particles of mesh sizes $\leq 0.1 \mathrm{~mm}$. A $5.0 \mathrm{~g}$ stool sample was extracted out with $25 \mathrm{~mL}$ hot deionized water $\left(50^{\circ} \mathrm{C}\right)$ for $6 \mathrm{hr}$ to measure the $\mathrm{F}^{-}$and $\mathrm{pH}$ values.

The first morning urine sample $(100 \mathrm{~mL})$ was collected in a plastic bottle containing $0.2 \mathrm{~g}$ EDTA. Total 20 (4 $\times 5$ ) urine samples of domestic animals (i.e. cattle, buffalo, sheep and goat) were collected in January, 2013. The samples was shipped to the laboratory in insulated container at about $4^{\circ} \mathrm{C}$ and stored at $-20^{\circ} \mathrm{C}$ until use.

\subsection{Analysis}

The Metrohm ion meter-781 was used for the measurement of $\mathrm{F}^{-}$by using TISAB-III buffer in 1:1 ratio. The

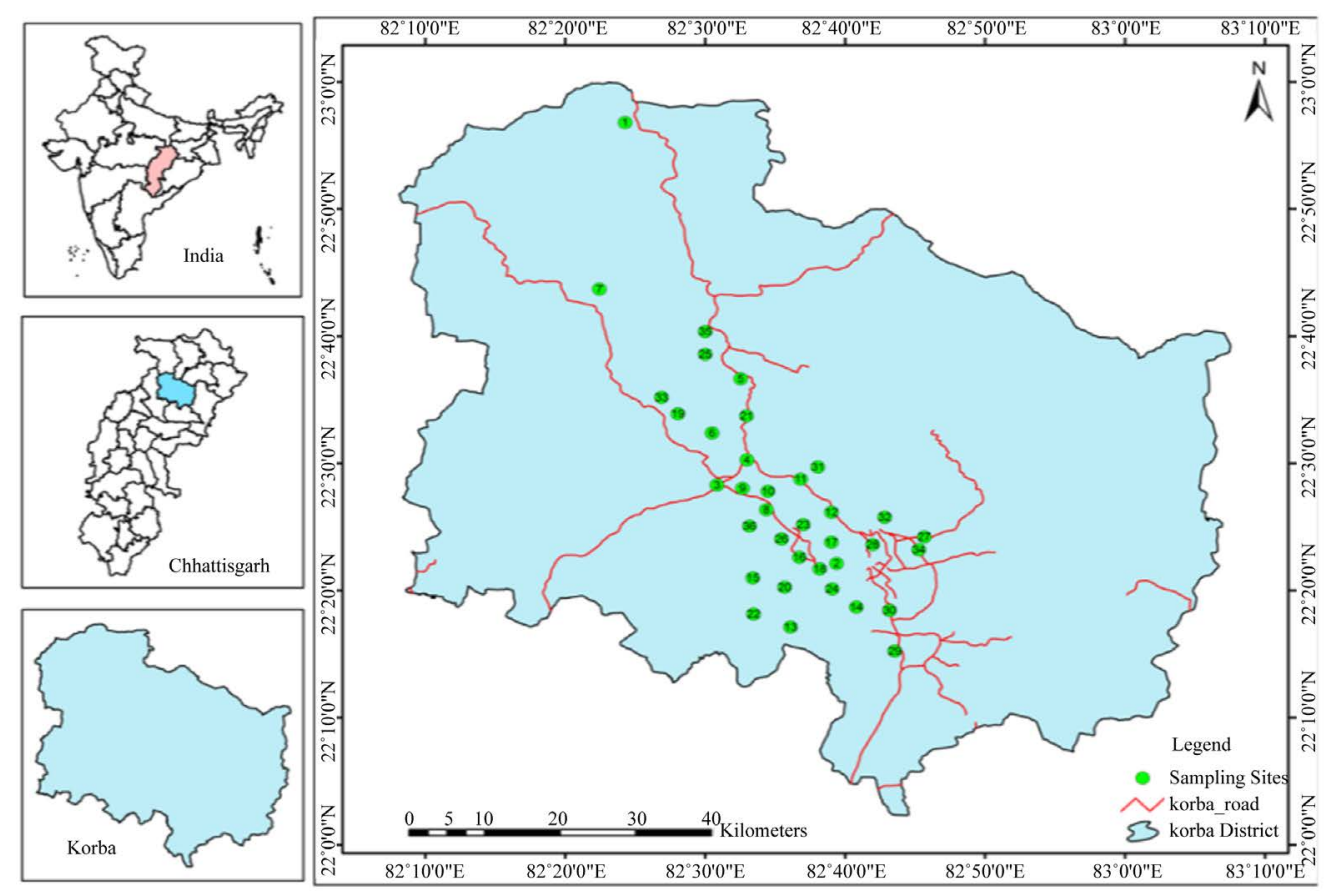

Figure 1. Representation of sampling locations in Korba basin. 
buffer was prepared by dissolving 300 g sodium citrate, 22 g 1,2-cyclohexanediamine-N,N,N,N-tetraaceticacid and $60 \mathrm{~g} \mathrm{NaCl}$ in a volume of 1-L with the de-ionized water with subsequent adjustment of pH value to 5.0 5.5). The Dionex ion chromatography-1100 was used for monitoring of ions i.e. $\mathrm{Cl}^{-}, \mathrm{NO}_{3}^{-}, \mathrm{SO}_{4}^{2-}, \mathrm{Na}^{+}, \mathrm{K}^{+}$, $\mathrm{Mg}^{2+}$ and $\mathrm{Ca}^{2+}$. The Thermos ICP-OES and ICP-MS (Polish Geological Institute, NRI, Central Chemical Laboratory, Warsaw) were employed for analysis of the elements.

\section{Results and Discussion}

\subsection{Geology and Hydrology}

The rock of the studied area was formed in the Archaean to Cenozoic ages. They consist of granite gneiss and granitoids covering with medium to coarse grained arkosic sandstone, a few pebble beds, conglomerate and shale of coal. The ground water occurs under phreatic, semi-confined and con-fined conditions control of the inter granular pore spaces in the shallow weathered zones and joints, fractures and caverns in deeper horizons. The water level depth is varied from 3.4 - 11.6 mbgl (meter below ground level) during the post-monsoon period in the aquifers.

\subsection{Physico-Chemical Characteristics of Groundwater}

The physico-chemical parameters of the groundwater are presented in Tables 1-3. The age and depth of 30 tube wells was ranged from $4.0-130 \mathrm{Yr}$ and $4-18 \mathrm{~m}$ with mean value of $7 \pm 1 \mathrm{Yr}$ and $11 \pm 1 \mathrm{~m}$, respectively. A slight variation in temperature of the groundwater was recorded, ranging from $29.5-31.2^{\circ} \mathrm{C}$ with mean value of $30.6 \pm 0.1^{\circ} \mathrm{C}$. The $\mathrm{pH}$ value of the water was varied from $5.9-8.2$ with mean value of $7.1 \pm 0.2$. The groundwater was found to be acidic in few locations (i.e. Surabahar, Sutara, Turali, Lalmatiya, Jenara, Dhelawdih, Banki Mongara, Dipka, Kusmunda, Bhilai, Hardibazar and Rumgara) due to the existence of higher content of the acids. The EC,TDS, DO and RP value of the groundwater was varied from 296 - $964 \mu \mathrm{S} / \mathrm{cm}, 414$ - $990 \mathrm{mg} / \mathrm{L}, 5.6$ $6.7 \mathrm{mg} / \mathrm{L}$ and $199-241 \mathrm{mV}$ with mean value of $497 \pm 56 \mu \mathrm{S} / \mathrm{cm}, 628 \pm 61 \mathrm{mg} / \mathrm{L}, 6.0 \pm 0.1 \mathrm{mg} / \mathrm{L}$ and $218 \pm 5$ $\mathrm{mV}$, respectively. The value of DO, EC and TDS was found moderately higher to the recommended value of 4.0 $\mathrm{mg} / \mathrm{L}, 300 \mu \mathrm{S} / \mathrm{cm}$ and $500 \mathrm{mg} / \mathrm{L}$, respectively [22] [23]. However, the RP value was observed to be at least 3-times less than the recommended value of $600 \mathrm{mV}$.

The concentration of $\mathrm{F}^{-}, \mathrm{Cl}^{-}, \mathrm{NO}_{3}^{-}, \mathrm{SO}_{4}^{2-}, \mathrm{Na}^{+}, \mathrm{K}^{+}, \mathrm{Mg}^{2+}, \mathrm{Ca}^{2+}, \mathrm{Al}, \mathrm{SiO}_{2}, \mathrm{Fe}, \mathrm{Mn}$ and $\mathrm{Zn}$ was ranged from 2.1 - 12, 11 - 92, 10 - 90, 10 - 72, 10 - 70, 0.6 - 8.7, 3.0 - 44, 8.0 - 78, 7.2 - 45, 9.0 - 70, 0.4 - 8.2, 0.2 - 1.5 and $1.0-2.4 \mathrm{mg} / \mathrm{L}$ with mean value of $5.5 \pm 0.9,34 \pm 7,28 \pm 7,21 \pm 5,24 \pm 6,3.7 \pm 0.8,11 \pm 3,31 \pm 6,16 \pm 3,31 \pm$ 6, $2.2 \pm 0.6,0.5 \pm 0.1$ and $1.5 \pm 0.1 \mathrm{mg} / \mathrm{L}$, respectively. Similarly, concentration of other metals: Li, Be, Rb, Co, $\mathrm{Ni}, \mathrm{Cu}, \mathrm{Sn}, \mathrm{Sb}, \mathrm{Mo}, \mathrm{Cd}, \mathrm{La}, \mathrm{Ce}, \mathrm{Pb}$ and U was observed in microgram levels, ranging from 4.7 - 36, 1.1 - 6.7, 1.4 - 38, 1.1 - 7.7, 2.4 - 27, 2.3 - 22, 1.2 - 11, 0.9 - 9.8, 0.1 - 5.0, 0.11 - 0.33, 0.1 - 2.1, $0.1-0.8,1.1-29$ and 1.0 $-12 \mu \mathrm{g} / \mathrm{L}$ with mean value of $16 \pm 3,2.1 \pm 0.5,16 \pm 5,3.3 \pm 0.8,9.2 \pm 2.4,8.0 \pm 2.1,3.0 \pm 1.0,3.2 \pm 0.8,1.0 \pm$ $0.4,0.19 \pm 0.02,0.4 \pm 0.2,0.4 \pm 0.1,7.0 \pm 2.4$ and $2.5 \pm 1.0 \mu \mathrm{g} / \mathrm{L}$, respectively. Among them, the highest content of $\mathrm{Cl}^{-}$was observed, may be due to leaching from the coal. The occurrence trend of 27 elements in the water was found in following decreasing order: $\mathrm{Cl}^{-}>\mathrm{Ca} \approx \mathrm{SiO}_{2}<\mathrm{NO}_{3}^{-}<\mathrm{Na}^{+}>\mathrm{SO}_{4}^{2-}>\mathrm{Al}>\mathrm{Mg}>\mathrm{K}^{+}>\mathrm{F}^{-}>$ $\mathrm{Fe}>\mathrm{Zn}>\mathrm{Mn}>>\mathrm{Li} \approx \mathrm{Rb}>\mathrm{Ni}>\mathrm{Cu}>\mathrm{Pb}>\mathrm{Sb} \approx \mathrm{Co}>\mathrm{Sn}>\mathrm{U}>\mathrm{Be}<\mathrm{Mo}>\mathrm{La} \approx \mathrm{Ce}>\mathrm{Cd}$. The concentration of $\mathrm{F}^{-}$in the groundwater of the studied area was found to be comparable to $\mathrm{F}^{-}$contents reported in the water of other locations of the country [5]-[18].

Factor analysis was used for 17 variables of the water. Four factors were extracted which explain $83.27 \%$ of the total variance. A 58.49\% of the total variance was explained by Factor-1, showing strong positive loadings on EC, TDS, $\mathrm{F}^{-}, \mathrm{Cl}^{-}, \mathrm{SO}_{4}^{2-}, \mathrm{Na}^{+}, \mathrm{Mg}^{2+}, \mathrm{Fe}, \mathrm{Mn}, \mathrm{Zn}, \mathrm{Be}, \mathrm{Rb}, \mathrm{Co}, \mathrm{Ni}, \mathrm{Cu}, \mathrm{Cd}, \mathrm{Pb}, \mathrm{La}, \mathrm{Ce}$ and Ca ${ }^{2+}$. They were related to the mineralization of the groundwater which involved also weathering of gypsum and fluoride bearing minerals such as $\mathrm{CaF}_{2}$. They were correlated well in the water as shown in Table 4, Table 5. A 10.93\% of the total variance was accounted by factor-2, pyrite with strong positive loadings on $\mathrm{NO}_{3}^{-}$and $\mathrm{K}^{+}$, related to coal burning and mining activities. A 7.78\% of the total variance was explained by factor-3, describing a strong loading on $\mathrm{SiO}_{2}$. A $6.06 \%$ of total variance was explained by factor-4 related to the age factor of tube wells.

\subsection{Physico-Chemical Characteristics of Surface Water}

The physico-chemical characteristic of the surface water is shown in Tables 6-8. The value of T, pH, DO, RP, 
Table 1. Physical characteristics of groundwater in Korba basin, January 2013.

\begin{tabular}{|c|c|c|c|c|c|c|}
\hline S. No. & Location & $\mathrm{pH}$ & $\mathrm{EC}, \mu \mathrm{S} / \mathrm{cm}$ & TDS, mg/L & $\mathrm{DO}, \mathrm{mg} / \mathrm{L}$ & $\mathrm{RP}, \mathrm{mV}$ \\
\hline 1 & Pauli & 7.4 & 296 & 745 & 5.9 & 199 \\
\hline 2 & Surabahar & 6.6 & 527 & 627 & 5.8 & 228 \\
\hline 3 & Sutara & 5.9 & 398 & 563 & 5.8 & 241 \\
\hline 4 & Katghora & 7.8 & 388 & 509 & 5.9 & 202 \\
\hline 5 & Konkona & 7.3 & 514 & 589 & 5.7 & 221 \\
\hline 6 & Turali & 6.6 & 964 & 813 & 6.2 & 230 \\
\hline 7 & Lalmatiya & 6.4 & 312 & 588 & 6.1 & 237 \\
\hline 8 & Jenara & 6.8 & 464 & 574 & 5.6 & 199 \\
\hline 9 & Lakhanpur & 7.1 & 633 & 611 & 5.8 & 215 \\
\hline 10 & Dhelawdih & 6.7 & 515 & 628 & 6.1 & 212 \\
\hline 11 & Churri & 7.2 & 629 & 738 & 6.7 & 201 \\
\hline 12 & Gopalpur & 7.7 & 519 & 922 & 5.9 & 241 \\
\hline 13 & Darri & 7.2 & 523 & 650 & 5.8 & 202 \\
\hline 14 & Banki Mongara & 6.8 & 634 & 722 & 5.8 & 221 \\
\hline 15 & Dipka & 6.9 & 511 & 441 & 5.9 & 230 \\
\hline 16 & Ghordeva & 7.2 & 534 & 487 & 5.7 & 199 \\
\hline 17 & Balgi & 7.0 & 713 & 933 & 6.2 & 228 \\
\hline 18 & Kuchana & 7.3 & 391 & 633 & 6.1 & 241 \\
\hline 19 & Kusmunda & 6.7 & 704 & 926 & 5.6 & 202 \\
\hline 20 & Bhilai & 6.8 & 362 & 455 & 5.8 & 221 \\
\hline 21 & Dadar & 7.0 & 322 & 464 & 6.1 & 230 \\
\hline 22 & Hardibazar & 6.9 & 367 & 434 & 6.7 & 237 \\
\hline 23 & NTPC & 7.4 & 380 & 471 & 5.7 & 199 \\
\hline 24 & Barpali & 7.6 & 342 & 414 & 6.1 & 215 \\
\hline 25 & Manikpur & 7.2 & 650 & 821 & 6.2 & 212 \\
\hline 26 & Korba & 7.3 & 756 & 990 & 6.1 & 201 \\
\hline 27 & Balco & 7.1 & 410 & 497 & 5.6 & 221 \\
\hline 28 & Rumgara & 6.9 & 390 & 449 & 5.8 & 230 \\
\hline 29 & Urga & 8.2 & 418 & 680 & 6.1 & 237 \\
\hline 30 & Bilaikhund & 7.8 & 347 & 462 & 6.3 & 199 \\
\hline
\end{tabular}


Table 2. Concentration of major elements in groundwater during January 2013, mg/L.

\begin{tabular}{|c|c|c|c|c|c|c|c|c|c|c|c|c|c|}
\hline S. No. & $\mathrm{F}^{-}$ & $\mathrm{Cl}^{-}$ & $\mathrm{NO}_{3}^{-}$ & $\mathrm{SO}_{4}^{2-}$ & $\mathrm{Na}^{+}$ & $\mathrm{K}^{+}$ & $\mathrm{Mg}^{2+}$ & $\mathrm{Ca}^{2+}$ & $\mathrm{Al}$ & $\mathrm{SiO}_{2}$ & $\mathrm{Fe}$ & Mn & $\mathrm{Zn}$ \\
\hline 1 & 2.8 & 32 & 28 & 14 & 11 & 6 & 16 & 52 & 17 & 64 & 3.7 & 0.51 & 1.4 \\
\hline 2 & 4.5 & 18 & 11 & 12 & 17 & 2 & 12 & 36 & 15 & 11 & 1.2 & 0.31 & 2.4 \\
\hline 3 & 3.7 & 39 & 17 & 12 & 12 & 5 & 6 & 18 & 14 & 23 & 2.0 & 0.55 & 2.2 \\
\hline 4 & 5.8 & 53 & 14 & 15 & 14 & 2 & 7 & 22 & 15 & 19 & 1.1 & 0.33 & 1.3 \\
\hline 5 & 10 & 39 & 35 & 14 & 22 & 2 & 9 & 30 & 9.1 & 23 & 1.0 & 0.17 & 2.0 \\
\hline 6 & 4.6 & 92 & 23 & 72 & 17 & 5 & 6 & 17 & 10 & 18 & 4.1 & 0.69 & 1.6 \\
\hline 7 & 3.5 & 32 & 21 & 24 & 23 & 6 & 16 & 51 & 7.2 & 9 & 1.1 & 0.38 & 1.7 \\
\hline 8 & 3.8 & 39 & 21 & 44 & 17 & 2 & 8 & 22 & 18 & 12 & 2.8 & 0.68 & 1.2 \\
\hline 9 & 3.8 & 39 & 30 & 22 & 28 & 2 & 9 & 24 & 16 & 26 & 1.1 & 0.93 & 2.0 \\
\hline 10 & 4.5 & 50 & 31 & 10 & 16 & 3 & 11 & 36 & 11 & 35 & 0.4 & 0.22 & 1.3 \\
\hline 11 & 6.0 & 67 & 31 & 26 & 18 & 3 & 11 & 36 & 11 & 35 & 0.4 & 0.22 & 1.3 \\
\hline 12 & 4.8 & 39 & 25 & 30 & 24 & 1.6 & 22 & 66 & 19 & 70 & 4.2 & 0.48 & 1.2 \\
\hline 13 & 3.6 & 11 & 10 & 13 & 18 & 1.3 & 5 & 22 & 17 & 12 & 1.4 & 0.29 & 2.0 \\
\hline 14 & 8.4 & 39 & 15 & 16 & 60 & 2.7 & 18 & 36 & 16 & 25 & 2.3 & 0.52 & 1.8 \\
\hline 15 & 11 & 18 & 13 & 10 & 16 & 1.5 & 5 & 32 & 17 & 21 & 1.2 & 0.31 & 1.1 \\
\hline 16 & 4.6 & 28 & 31 & 15 & 10 & 5.9 & 8 & 22 & 10 & 25 & 1.1 & 0.16 & 1.7 \\
\hline 17 & 5.8 & 67 & 90 & 40 & 38 & 3.3 & 14 & 48 & 45 & 20 & 4.7 & 0.65 & 1.3 \\
\hline 18 & 3.7 & 11 & 19 & 13 & 15 & 4.8 & 6 & 18 & 7.5 & 10 & 1.2 & 0.36 & 1.4 \\
\hline 19 & 7.9 & 43 & 19 & 20 & 53 & 1.3 & 26 & 78 & 21 & 68 & 3.2 & 0.64 & 1.0 \\
\hline 20 & 6.2 & 14 & 27 & 14 & 18 & 8.5 & 3 & 10 & 18 & 29 & 1.3 & 0.88 & 1.7 \\
\hline 21 & 2.9 & 14 & 28 & 14 & 17 & 3.9 & 4 & 18 & 12 & 39 & 0.5 & 0.21 & 1.1 \\
\hline 22 & 2.9 & 14 & 28 & 14 & 17 & 3.9 & 4 & 8 & 12 & 39 & 0.5 & 0.21 & 1.1 \\
\hline 23 & 2.1 & 21 & 13 & 16 & 10 & 1.7 & 8 & 10 & 30 & 42 & 1.4 & 0.35 & 1.3 \\
\hline 24 & 3.5 & 14 & 15 & 12 & 14 & 3.3 & 4 & 14 & 26 & 29 & 1.7 & 0.35 & 1.1 \\
\hline 25 & 12 & 38 & 83 & 50 & 65 & 3.9 & 44 & 60 & 18 & 55 & 8.2 & 1.53 & 1.8 \\
\hline 26 & 7.3 & 70 & 78 & 40 & 58 & 3.9 & 16 & 50 & 11 & 58 & 5.9 & 0.86 & 1.2 \\
\hline 27 & 7.7 & 28 & 31 & 15 & 10 & 5.9 & 8 & 22 & 10 & 25 & 1.3 & 0.16 & 1.7 \\
\hline 28 & 3.5 & 14 & 18 & 16 & 11 & 6.7 & 6 & 24 & 7.6 & 40 & 1.7 & 0.27 & 1.0 \\
\hline 29 & 9.2 & 18 & 17 & 10 & 70 & 0.6 & 12 & 36 & 27 & 23 & 1.8 & 0.32 & 1.8 \\
\hline 30 & 3.9 & 18 & 19 & 15 & 10 & 8.7 & 5 & 16 & 24 & 31 & 2.1 & 0.35 & 1.0 \\
\hline
\end{tabular}


Table 3. Concentration of trace elements in groundwater during January 2013, $\mu \mathrm{g} / \mathrm{mL}$.

\begin{tabular}{|c|c|c|c|c|c|c|c|c|c|c|c|c|c|c|}
\hline S. No. & $\mathrm{Li}$ & Be & $\mathrm{Rb}$ & Co & $\mathrm{Ni}$ & $\mathrm{Cu}$ & Sn & $\mathrm{Sb}$ & Mo & $\mathrm{Cd}$ & $\mathrm{La}$ & Ce & $\mathrm{Pb}$ & $\mathrm{U}$ \\
\hline 1 & 18 & 2.4 & 3.8 & 1.9 & 7.4 & 6.5 & 1.6 & 2.3 & 0.66 & 0.25 & 0.24 & 0.51 & 4.7 & 2.8 \\
\hline 2 & 20 & 2.4 & 37 & 2.9 & 13.3 & 4.8 & 3.4 & 4.7 & 1.76 & 0.13 & 0.22 & 0.23 & 2.7 & 1.6 \\
\hline 3 & 18 & 2.5 & 3.2 & 2.2 & 10.6 & 3.0 & 2.7 & 3.4 & 2.08 & 0.22 & 0.15 & 0.20 & 1.2 & 1.4 \\
\hline 4 & 17 & 1.5 & 4.9 & 1.7 & 5.1 & 8.6 & 1.7 & 2.8 & 2.05 & 0.14 & 0.52 & 0.71 & 5.6 & 1.4 \\
\hline 5 & 12 & 1.5 & 25.5 & 5.0 & 3.9 & 8.8 & 2.0 & 6.0 & 0.28 & 0.17 & 0.27 & 0.08 & 9.0 & 1.3 \\
\hline 6 & 11 & 1.5 & 8.3 & 4.2 & 10.0 & 4.1 & 2.6 & 4.6 & 2.10 & 0.13 & 0.24 & 0.31 & 1.9 & 1.6 \\
\hline 7 & 9 & 1.5 & 21.1 & 3.0 & 9.8 & 4.7 & 2.2 & 3.3 & 1.11 & 0.20 & 0.56 & 0.67 & 7.3 & 1.6 \\
\hline 8 & 19 & 1.8 & 2.2 & 1.4 & 2.9 & 2.9 & 1.5 & 1.0 & 0.65 & 0.19 & 0.20 & 0.13 & 4.3 & 3.8 \\
\hline 9 & 36 & 2.2 & 33 & 7.6 & 11.4 & 6.0 & 2.9 & 9.8 & 1.20 & 0.20 & 0.41 & 0.70 & 3.6 & 1.4 \\
\hline 10 & 11 & 5.2 & 38 & 7.7 & 26.6 & 18 & 11 & 1.7 & 0.06 & 0.33 & 2.11 & 0.80 & 9.6 & 1.6 \\
\hline 11 & 11 & 5.2 & 28 & 7.7 & 23.8 & 18 & 10 & 1.9 & 0.08 & 0.33 & 1.11 & 0.60 & 9.1 & 1.6 \\
\hline 12 & 16 & 1.7 & 3.4 & 1.6 & 6.1 & 5.1 & 1.3 & 2.1 & 0.56 & 0.21 & 0.19 & 0.44 & 4.3 & 2.1 \\
\hline 13 & 18 & 1.7 & 34 & 2.4 & 11 & 3.8 & 2.8 & 4.2 & 1.49 & 0.11 & 0.17 & 0.2 & 2.5 & 1.2 \\
\hline 14 & 16 & 1.8 & 2.9 & 1.8 & 8.8 & 2.4 & 2.2 & 3.1 & 1.76 & 0.19 & 0.12 & 0.17 & 1.1 & 1.1 \\
\hline 15 & 15 & 1.1 & 4.4 & 1.4 & 4.2 & 6.8 & 1.4 & 2.5 & 1.74 & 0.12 & 0.41 & 0.61 & 5.1 & 1.1 \\
\hline 16 & 11 & 1.1 & 23 & 4.2 & 3.2 & 6.9 & 1.6 & 5.4 & 0.24 & 0.14 & 0.21 & 0.07 & 8.3 & 1.0 \\
\hline 17 & 10 & 1.1 & 7.5 & 3.5 & 8.3 & 3.2 & 2.1 & 4.1 & 1.78 & 0.11 & 0.19 & 0.27 & 1.7 & 1.2 \\
\hline 18 & 8.6 & 1.1 & 19 & 2.5 & 8.1 & 3.7 & 1.8 & 3.0 & 0.94 & 0.17 & 0.44 & 0.58 & 6.7 & 1.2 \\
\hline 19 & 17 & 1.3 & 2 & 1.2 & 2.4 & 2.3 & 1.2 & 0.9 & 0.55 & 0.16 & 0.16 & 0.11 & 3.9 & 2.9 \\
\hline 20 & 33 & 1.6 & 30 & 6.3 & 9.4 & 4.7 & 2.4 & 8.8 & 1.02 & 0.17 & 0.32 & 0.61 & 3.3 & 1.1 \\
\hline 21 & 10 & 3.7 & 35 & 6.4 & 22 & 14 & 9.1 & 1.5 & 0.05 & 0.28 & 1.66 & 0.69 & 27 & 1.2 \\
\hline 22 & 10 & 3.7 & 35 & 6.4 & 23 & 14 & 9.1 & 1.5 & 0.05 & 0.28 & 1.66 & 0.69 & 29 & 1.2 \\
\hline 23 & 29 & 1.9 & 1.4 & 1.2 & 2.4 & 2.3 & 1.6 & 1.1 & 0.87 & 0.19 & 0.14 & 0.22 & 2.6 & 10 \\
\hline 24 & 13 & 1.5 & 28 & 1.9 & 7.7 & 18 & 1.7 & 1.2 & 0.24 & 0.25 & 0.19 & 0.31 & 20 & 1.1 \\
\hline 25 & 21 & 1.3 & 1.5 & 1.6 & 5.5 & 9.0 & 1.8 & 1.1 & 0.55 & 0.17 & 0.11 & 0.17 & 4.9 & 4.1 \\
\hline 26 & 25 & 6.7 & 13 & 1.3 & 2.7 & 5.7 & 1.7 & 1.2 & 4.95 & 0.11 & 0.12 & 0.23 & 5.2 & 9.0 \\
\hline 27 & 6.1 & 1.1 & 23 & 4.2 & 3.2 & 6.9 & 1.6 & 5.4 & 0.24 & 0.19 & 0.21 & 0.27 & 8.3 & 1.0 \\
\hline 28 & 4.7 & 1.1 & 1.5 & 2.9 & 10 & 22 & 2.4 & 3.2 & 1.23 & 0.16 & 0.14 & 0.25 & 6.8 & 12 \\
\hline 29 & 20 & 1.2 & 7.3 & 1.1 & 7.3 & 21 & 1.2 & 1.2 & 0.22 & 0.31 & 0.31 & 0.58 & 8.4 & 1.2 \\
\hline 30 & 9.2 & 1.1 & 1.4 & 1.1 & 5.4 & 3.8 & 1.5 & 1.9 & 0.55 & 0.16 & 0.13 & 0.19 & 1.5 & 1.2 \\
\hline
\end{tabular}


Table 4. Correlation matrix of elements in groundwater.

\begin{tabular}{|c|c|c|c|c|c|c|c|c|c|c|c|c|c|}
\hline & $\mathrm{F}^{-}$ & $\mathrm{Cl}^{-}$ & $\mathrm{NO}_{3}^{-}$ & $\mathrm{SO}_{4}^{2-}$ & $\mathrm{Na}^{+}$ & $\mathrm{K}^{+}$ & $\mathrm{Mg}^{2+}$ & $\mathrm{Ca}^{2+}$ & $\mathrm{Al}$ & $\mathrm{SiO}_{2}$ & $\mathrm{Fe}$ & Mn & $\mathrm{Zn}$ \\
\hline $\mathrm{F}^{-}$ & 1 & & & & & & & & & & & & \\
\hline $\mathrm{Cl}^{-}$ & 0.18 & 1 & & & & & & & & & & & \\
\hline $\mathrm{NO}_{3}^{-}$ & 0.36 & 0.47 & 1 & & & & & & & & & & \\
\hline $\mathrm{SO}_{4}^{2-}$ & 0.13 & 0.72 & 0.52 & 1 & & & & & & & & & \\
\hline $\mathrm{Na}^{+}$ & 0.64 & 0.24 & 0.46 & 0.28 & 1 & & & & & & & & \\
\hline $\mathrm{K}^{+}$ & -0.26 & -0.12 & 0.09 & 0.01 & -0.36 & 1 & & & & & & & \\
\hline $\mathrm{Mg}^{2+}$ & 0.51 & 0.27 & 0.52 & 0.39 & 0.68 & -0.20 & 1 & & & & & & \\
\hline $\mathrm{Ca}^{2+}$ & 0.42 & 0.34 & 0.40 & 0.25 & 0.59 & -0.28 & 0.83 & 1 & & & & & \\
\hline $\mathrm{Al}$ & 0.04 & 0.01 & 0.26 & 0.07 & 0.26 & -0.25 & 0.13 & 0.13 & 1 & & & & \\
\hline $\mathrm{SiO}_{2}$ & 0.10 & 0.12 & 0.30 & 0.10 & 0.29 & -0.01 & 0.54 & 0.56 & 0.09 & 1 & & & \\
\hline $\mathrm{Fe}$ & 0.37 & 0.44 & 0.69 & 0.70 & 0.57 & 0.01 & 0.74 & 0.58 & 0.29 & 0.48 & 1 & & \\
\hline Mn & 0.32 & 0.32 & 0.56 & 0.61 & 0.54 & 0.05 & 0.63 & 0.35 & 0.21 & 0.29 & 0.78 & 1 & \\
\hline $\mathrm{Zn}$ & 0.16 & -0.02 & -0.06 & -0.06 & 0.12 & -0.10 & 0.09 & -0.06 & -0.16 & -0.44 & -0.05 & 0.14 & 1 \\
\hline
\end{tabular}

Table 5. Correlation matrix of trace elements in groundwater.

\begin{tabular}{|c|c|c|c|c|c|c|c|c|c|c|c|c|c|c|}
\hline & $\mathrm{Li}$ & $\mathrm{Be}$ & $\mathrm{Rb}$ & Co & $\mathrm{Ni}$ & $\mathrm{Cu}$ & Sn & $\mathrm{Sb}$ & Mo & $\mathrm{Cd}$ & $\mathrm{La}$ & Ce & $\mathrm{Pb}$ & $\mathrm{U}$ \\
\hline $\mathrm{Li}$ & 1 & & & & & & & & & & & & & \\
\hline $\mathrm{Be}$ & 0.11 & 1 & & & & & & & & & & & & \\
\hline $\mathrm{Rb}$ & 0.00 & 0.38 & 1 & & & & & & & & & & & \\
\hline Co & -0.04 & 0.42 & 0.75 & 1 & & & & & & & & & & \\
\hline $\mathrm{Ni}$ & -0.22 & 0.57 & 0.63 & 0.74 & 1 & & & & & & & & & \\
\hline $\mathrm{Cu}$ & -0.31 & 0.28 & 0.29 & 0.35 & 0.51 & 1 & & & & & & & & \\
\hline Sn & -0.24 & 0.68 & 0.61 & 0.76 & 0.95 & 0.51 & 1 & & & & & & & \\
\hline $\mathrm{Sb}$ & 0.32 & -0.25 & 0.39 & 0.48 & -0.05 & -0.25 & -0.15 & 1 & & & & & & \\
\hline Mo & 0.30 & 0.27 & -0.25 & -0.33 & -0.29 & -0.39 & -0.32 & 0.07 & 1 & & & & & \\
\hline Cd & -0.12 & 0.41 & 0.30 & 0.44 & 0.65 & 0.64 & 0.66 & -0.31 & -0.60 & 1 & & & & \\
\hline $\mathrm{La}$ & -0.26 & 0.58 & 0.58 & 0.69 & 0.86 & 0.50 & 0.93 & -0.19 & -0.37 & 0.67 & 1 & & & \\
\hline Ce & 0.04 & 0.30 & 0.39 & 0.49 & 0.59 & 0.39 & 0.53 & 0.05 & -0.17 & 0.52 & 0.69 & 1 & & \\
\hline $\mathrm{Pb}$ & -0.34 & 0.31 & 0.51 & 0.41 & 0.52 & 0.59 & 0.59 & -0.27 & -0.44 & 0.55 & 0.67 & 0.40 & 1 & \\
\hline $\mathrm{U}$ & 0.16 & 0.18 & -0.38 & -0.29 & -0.24 & 0.12 & -0.16 & -0.28 & 0.34 & -0.18 & -0.23 & -0.28 & -0.15 & 1 \\
\hline
\end{tabular}

Table 6. Physical parameter of surface water during January 2013.

\begin{tabular}{ccccccc}
\hline S. No. & Location & $\mathrm{pH}$ & $\mathrm{EC}, \mu \mathrm{S} / \mathrm{cm}$ & $\mathrm{TDS}, \mathrm{mg} / \mathrm{L}$ & $\mathrm{DO}, \mathrm{mg} / \mathrm{L}$ & $\mathrm{RP}, \mathrm{mV}$ \\
\hline 1 & Churri & 6.7 & 220 & 430 & 7.8 & 182 \\
2 & Rumgara & 7.2 & 331 & 409 & 6.7 & 202 \\
3 & Kusmunda & 7.5 & 493 & 413 & 5.8 & 210 \\
4 & Balco & 7.3 & 172 & 391 & 5.9 & 208 \\
5 & Manikpur & 7.4 & 210 & 494 & 8.1 & 176 \\
6 & Krishi Nagar & 6.8 & 304 & 437 & 7.8 & 182 \\
\hline
\end{tabular}


Table 7. Chemical parameter of surface water during January 2013.

\begin{tabular}{ccccccccccccccc}
\hline S. No. & $\mathrm{F}^{-}$ & $\mathrm{Cl}^{-}$ & $\mathrm{NO}_{3}^{-}$ & $\mathrm{SO}_{4}^{2-}$ & $\mathrm{Na}^{+}$ & $\mathrm{K}^{+}$ & $\mathrm{Mg}^{2+}$ & $\mathrm{Ca}^{2+}$ & $\mathrm{Al}$ & $\mathrm{SiO}_{2}$ & $\mathrm{Fe}$ & $\mathrm{Mn}$ & $\mathrm{Zn}$ \\
\hline 1 & 2.3 & 21 & 38 & 20 & 11 & 8.3 & 8.1 & 20 & 1.0 & 7.7 & 0.9 & 0.4 & 0.28 \\
2 & 1.8 & 11 & 17 & 10 & 46 & 15 & 5.2 & 16 & 1.2 & 7.7 & 0.4 & 0.4 & 0.39 \\
3 & 2.4 & 14 & 13 & 16 & 47 & 13 & 6.0 & 16 & 1.3 & 3.1 & 0.6 & 0.3 & 0.45 \\
4 & 2.2 & 18 & 17 & 30 & 16 & 7.1 & 5.1 & 18 & 1.4 & 9.2 & 1.3 & 0.7 & 0.29 \\
5 & 3.1 & 27 & 25 & 40 & 20 & 6.0 & 6.2 & 20 & 1.6 & 7.7 & 1.4 & 1.1 & 0.31 \\
6 & 2.6 & 14 & 30 & 20 & 18 & 9.2 & 9.1 & 28 & 1.3 & 6.1 & 1.5 & 1.2 & 0.42 \\
\hline
\end{tabular}

Table 8. Concentration of trace elements in surface water during January 2013.

\begin{tabular}{cccccccccccc}
\hline S. No. & $\mathrm{Li}$ & $\mathrm{Rb}$ & $\mathrm{Cr}$ & $\mathrm{Co}$ & $\mathrm{Ni}$ & $\mathrm{Cu}$ & $\mathrm{Sb}$ & $\mathrm{Pb}$ & $\mathrm{U}$ \\
\hline 1 & 16 & 13 & 5.1 & 2.7 & 5.2 & 6.6 & 3.8 & 7.4 & 0.92 \\
2 & 22 & 25 & 4.2 & 3.2 & 5.8 & 9.6 & 3.4 & 6.3 & 0.74 \\
3 & 23 & 18 & 4.6 & 3.9 & 4.8 & 7.5 & 3.4 & 5.7 & 0.89 \\
4 & 15 & 19 & 4.4 & 4.2 & 6.4 & 7.9 & 4.4 & 6.2 & 0.97 \\
5 & 14 & 14 & 4.8 & 2.5 & 4.0 & 6.4 & 2.4 & 4.5 & 0.68 \\
6 & 18 & 16 & 8.1 & 5.9 & 7.9 & 14 & 1.4 & 2.8 & 0.32 \\
\hline
\end{tabular}

EC and TDS of water $(n=6)$ in the post monsoon period was ranged from $20.8^{\circ} \mathrm{C}-22.2^{\circ} \mathrm{C}, 6.7-7.5,5.8-8.1$ $\mathrm{mg} / \mathrm{L}, 176-210 \mathrm{mV}, 172-493 \mu \mathrm{S} / \mathrm{cm}$ and $391-494 \mathrm{mg} / \mathrm{L}$ with mean value of $21.6^{\circ} \mathrm{C} \pm 0.4^{\circ} \mathrm{C}, 7.3 \pm 0.3,7.0 \pm$ $0.8 \mathrm{mg} / \mathrm{L}, 193 \pm 12 \mathrm{mV}, 429 \pm 29 \mu \mathrm{S} / \mathrm{cm}$ and $429 \pm 29 \mathrm{mg} / \mathrm{L}$, respectively. The concentration of $\mathrm{F}^{-}, \mathrm{Cl}^{-}, \mathrm{NO}_{3}^{-}$, $\mathrm{SO}_{4}^{2-}, \mathrm{Na}^{+}, \mathrm{K}^{+}, \mathrm{Mg}^{2+}, \mathrm{Ca}^{2+}, \mathrm{Al}, \mathrm{SiO}_{2}, \mathrm{Fe}, \mathrm{Mn}$ and $\mathrm{Zn}$ was ranged from 1.8 - 3.1, 11 - 27, 13 - 38, 10 - 40, 11 - 47, 6.0 - 15, 5.0 - 9.0, 16 - 28, 3.1 - 9.2, $0.06-0.26,0.4-1.5,0.3-1.2$ and $0.3-0.5 \mathrm{mg} / \mathrm{L}$ with mean value of $2.4 \pm$ $0.3,18 \pm 4.7,23 \pm 8,23 \pm 9,26 \pm 13,10 \pm 3,6.5 \pm 1.3,20 \pm 4,1.3 \pm 0.2,6.9 \pm 1.7,1.0 \pm 0.4,0.7 \pm 0.3$ and $0.4 \pm$ $0.1 \mathrm{mg} / \mathrm{L}$, respectively. Similarly, concentration of other metals: $\mathrm{Li}, \mathrm{Rb}, \mathrm{Cr}, \mathrm{Co}, \mathrm{Ni}, \mathrm{Cu}, \mathrm{Sb}, \mathrm{Pb}$ and $\mathrm{U}$ was observed at microgram levels, ranging from 14 - 23, 13 - 25, 4.2 - 8.1, 2.5 - 5.9, 4.0 - 7.9, 6.4 - 14, 1.4 - 4.4, 2.8 7.4 and $0.3-1.0 \mu \mathrm{g} / \mathrm{L}$ with mean value of $18 \pm 3,18 \pm 4,5.2 \pm 1.2,3.7 \pm 0.9,5.7 \pm 1.1,8.7 \pm 2.3,3.1 \pm 0.9,5.5$ \pm 1.3 and $0.8 \pm 0.2 \mu \mathrm{g} / \mathrm{L}$, respectively. They were found to occur in following decreasing order: $\mathrm{NO}_{3}^{-}>\mathrm{Ca}>$ $\mathrm{Na}^{+}>\mathrm{Cl}^{-}>\mathrm{SO}_{4}^{2-}>\mathrm{K}^{+}>\mathrm{Mg}>\mathrm{F}^{-}>\mathrm{Al}>\mathrm{Fe}>\mathrm{Mn}>\mathrm{Zn}>\mathrm{Li}>\mathrm{Rb}>\mathrm{Cu}>\mathrm{Pb}>\mathrm{Ni}>\mathrm{Cr}>\mathrm{Co}>\mathrm{Sb}>\mathrm{U}$. The concentration of $\mathrm{F}^{-}$in the surface water of the studied basin was found to be higher than other locations of the country [19] [20].

The factor analysis was applied and four factors were extracted. Factor- 1 was accounted for $48.70 \%$ of the total variance, related to anions, $\mathrm{Ca}^{2+}$ and $\mathrm{Mg}^{2+}$. It could be correlated to complex processes such as weathering of fluoride bearing minerals $\left(\mathrm{AlF}_{3}, \mathrm{CaF}_{2}, \mathrm{MgF}_{2}\right)$, gypsum, carbonate minerals and anthropogenic activities i.e. coal burning and leaching, aluminum plant effluents, etc. Factor- 2 was accounted for $22.15 \%$ of the total variance, related to EC, TDS and $\mathrm{Na}^{+}$which determined the mineralization of pond water. Factor-3 was accounted for $16.23 \%$ of the total variance having strong loadings of Fe and Mn, related to the speciation of Fe and Mn in the water. Factor- 4 was accounted for $9.23 \%$ of the total variance, negatively loaded with the $\mathrm{pH}$ values. This factor was in the inverse relationship with the other factors.

\subsection{Water Quality}

The concentration of $\mathrm{F}^{-}, \mathrm{Al}, \mathrm{Mn}$ and $\mathrm{Fe}$ was found to be several folds higher than recommended value of 1.0, $0.03,0.10$ and $0.30 \mathrm{mg} / \mathrm{L}$, respectively. The higher values of EC, TDS, RP, $\mathrm{F}^{-}, \mathrm{Cl}^{-}, \mathrm{NO}_{3}^{-}, \mathrm{Mg}^{2+}, \mathrm{Ca}^{2+}, \mathrm{SiO}_{2}, \mathrm{Al}$, $\mathrm{Fe}, \mathrm{Ni}, \mathrm{Zn}, \mathrm{Sb}, \mathrm{Pb}$ and $\mathrm{U}$ in the groundwater than the surface water was observed, may be due to leaching from the coal (Figure 2, Figure 3). The main sources of the contaminants in the water of the studied area expected are 


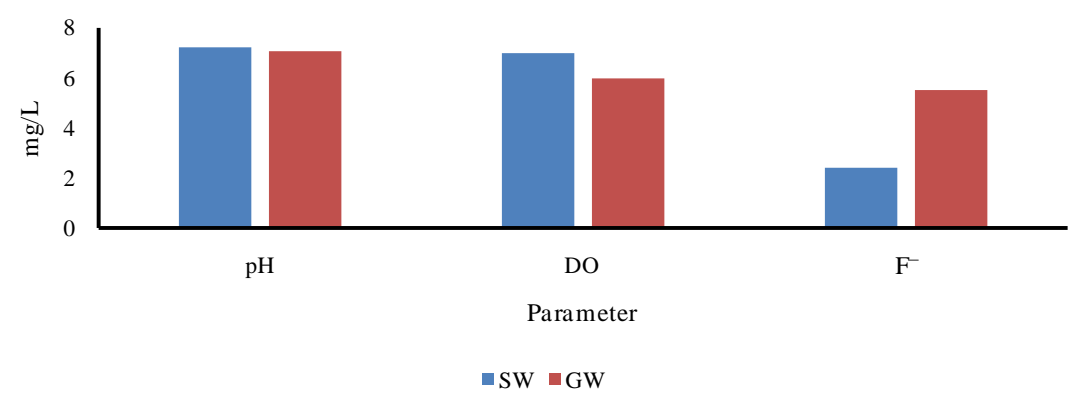

(a)

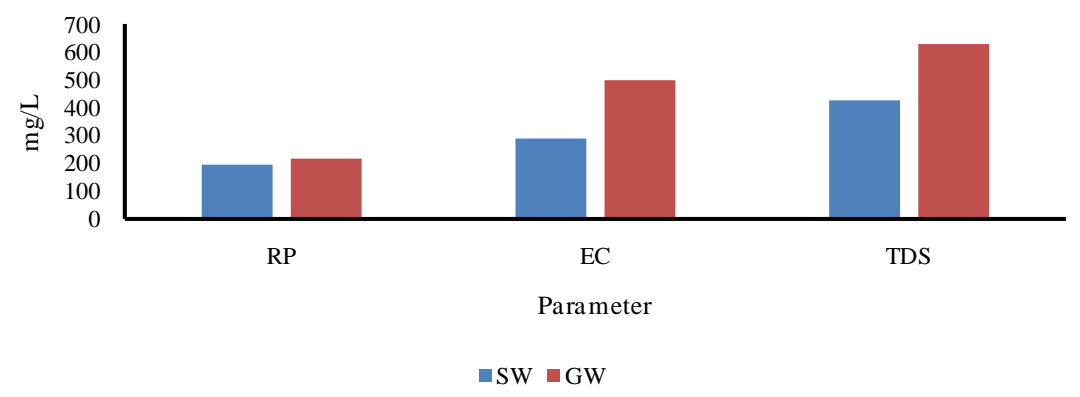

(b)

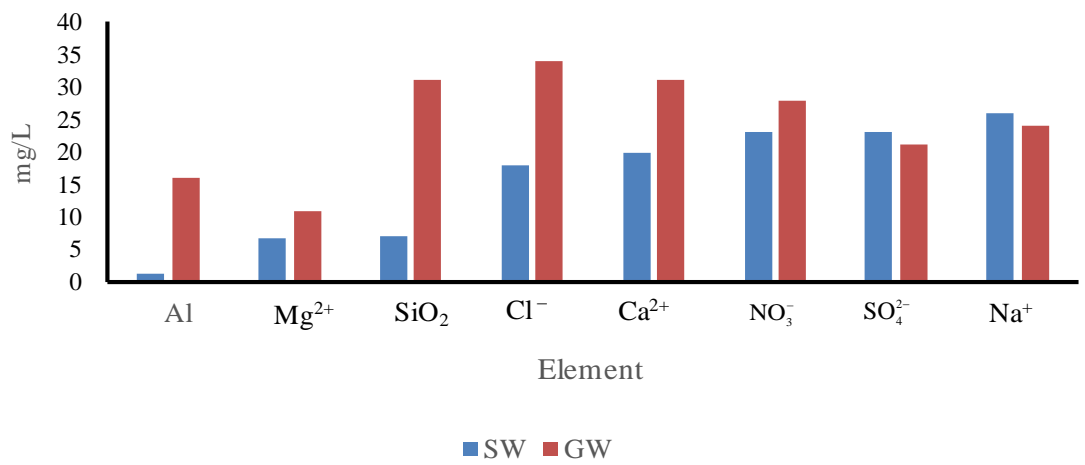

(c)

Figure 2. Distribution of $\mathrm{pH}$ and elements in ground and surface water in the post monsoon period, January, 2013.

coal mine leachates and the Aluminum and Thermal power plant effluents discharged into the environment.

\subsection{Exposure Assessment}

The toxic elements i.e. $\mathrm{F}^{-}$, $\mathrm{Al}, \mathrm{Mn}, \mathrm{Fe}, \mathrm{Cu}, \mathrm{Cd}, \mathrm{Pb}$ and $\mathrm{U}$ are exposed to human and animals through the contaminated water. Among them, the concentration of $\mathrm{F}^{-}$is dominated in ground and surface water. The stool and urine samples were reported as good indicator for the exposure assessment. In this study, the $\mathrm{F}^{-}$content was analyzed in stool and urine samples of domestic animals i.e. cattle, buffalo, sheep and goat (Table 9). The $\mathrm{F}^{-}$ concentration in the urine and stool samples $(5 \times 4 \times 2=40)$ was ranged from $32-63 \mathrm{mg} / \mathrm{L}$ and $186-356$ $\mathrm{mg} / \mathrm{kg}$ with mean value of $44 \mathrm{mg} / \mathrm{L}$ and $266 \mathrm{mg} / \mathrm{kg}$ (dried mass), respectively. The highest $\mathrm{F}^{-}$concentration was observed in the goat clinical samples, which might be due to higher intake of the contaminated biomass and water (Figure 4). Several cases of fluorosis diseases in the domestic animals of the basin was observed and shown in Figure 5.

\section{Conclusion}

The water of the Korba basin is contaminated with elements (i.e. $\mathrm{F}^{-}$, Al, Fe and Mn) by multiple sources (i.e. coal 


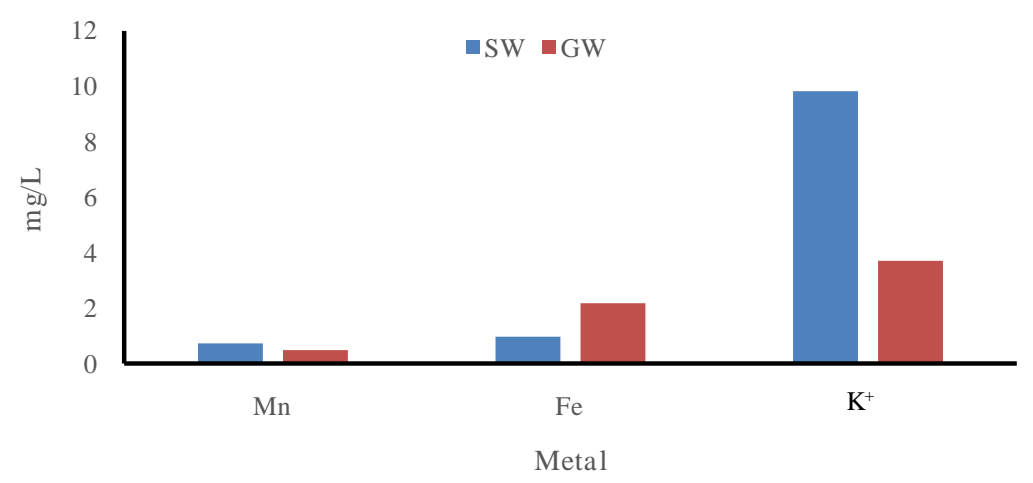

(a)

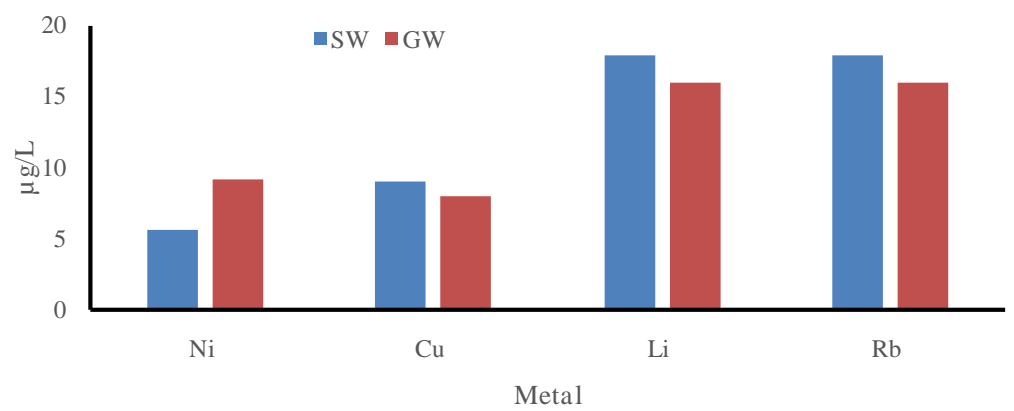

(b)

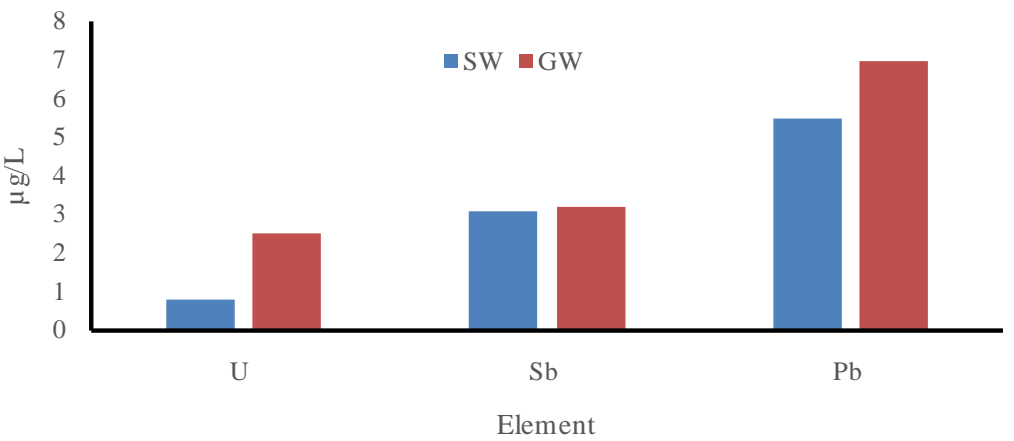

(c)

Figure 3. Distribution trace elementsin ground and surface water in the post monsoon period, January, 2013.

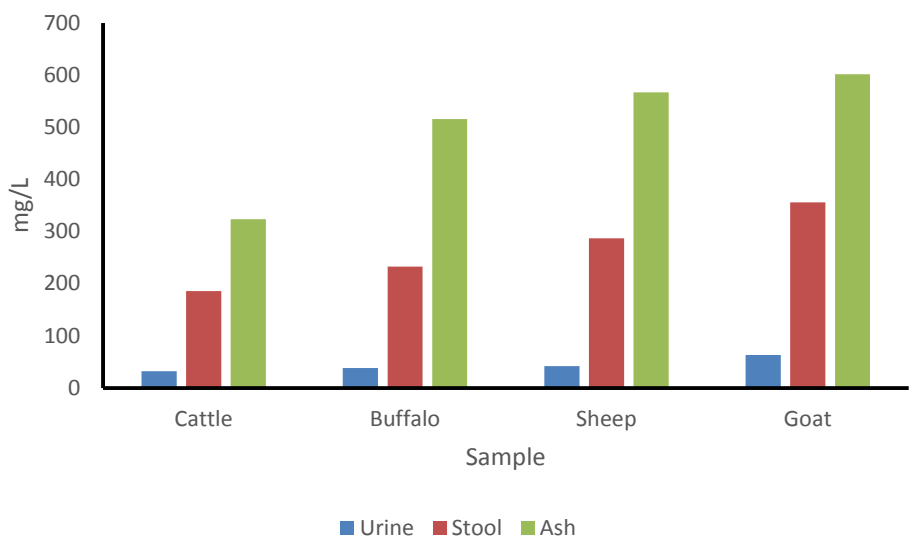

Figure 4. Comparison of $\mathrm{F}^{-}$concentration in clinical samples. 


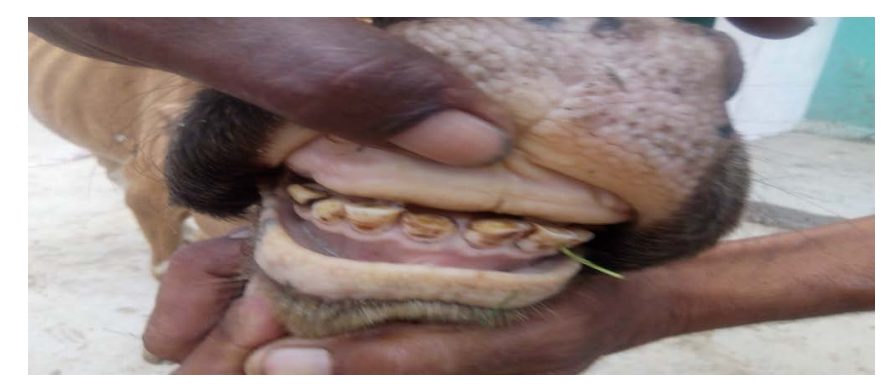

(a)

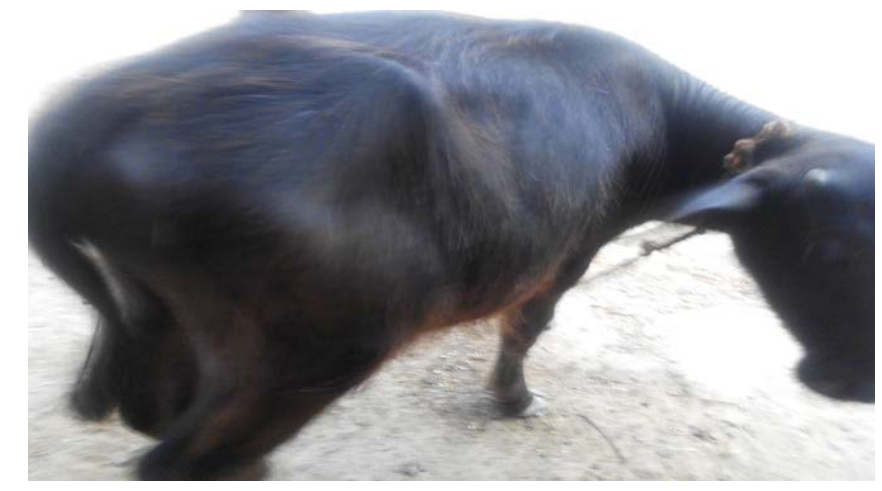

(b)

Figure 5. Dental (a) and hair (b) fluorosis in buffalo.

Table 9. Concentration of $\mathrm{F}^{-}$in clinical samples during January 2013.

\begin{tabular}{cccccc}
\hline S. No. & Sample & Cattle & Buffalo & Sheep & Goat \\
\hline 1 & Urine & 32 & 38 & 42 & 63 \\
2 & Stool & 186 & 233 & 287 & 356 \\
3 & Ash & 324 & 516 & 567 & 602 \\
\hline
\end{tabular}

burning, Aluminum plant effluent, mine leachate, etc.). Fluoride is enriched and several folds higher in the animal urines than recommended limit of $4 \mathrm{mg} / \mathrm{L}$ with higher prevalence of fluorosis diseases. The domestic animals are severely affected with fluorosis diseases due to higher consumption of the contaminated food and water.

\section{Acknowledgements}

We are thankful to the UGC, New Delhi for award of the Rajiv Gandhi Research Fellowship to KPR and SR.

\section{References}

[1] Finkelman, R.B. (1999) Trace Elements in Coal Environmental and Health Significance. Biological Trace Element Research, 67, 197-204. http://dx.doi.org/10.1007/BF02784420

[2] Ghosh, R., Majumder, T. and Ghosh, D.N. (1987) A Study of Trace Elements in Litho types of Some Selected Indian Coals. International Journal of Coal Geology, 8, 269-278. http://dx.doi.org/10.1016/0166-5162(87)90035-8

[3] Wu, D., Zheng, B., Tang, X., Li, S., Wang, B. and Wang, M. (2004) Fluorine in Chinese Coals. Fluoride, 37, $125-132$. http://www.fluorideresearch.org/372/files/FJ2004_v37_n2_p125-132.pdf

[4] Pandey, P.K., Pandey, M. and Chakraborty, M. (2013) Fluoride Mobilization due to Coal Mining in Parts of Chhattisgarh. Journal of Environmental Protection, 4, 385-388. http://dx.doi.org/10.4236/jep.2013.44046

[5] Gupta, S., Mondal, D. and Bardhan, A. (2012) Geochemical Provenance and Spatial Distribution of Fluoride in Groundwater in parts of Raniganj Coal Field, West Bengal, India. Archives of Applied Science Research, 4, 292-306. 
http://scholarsresearchlibrary.com/aasr-vol4-iss1/AASR-2012-4-1-292-306.pdf

[6] Borah, J. (2011) Monitoring Fluoride Concentration and Some other Physico-Chemical Properties of Groundwater of Tinsukia District, Assam, India. International Journal of ChemTech Research, 3, 1339-1342.

[7] Reza, R. and Singh, G. (2013) Groundwater Quality Status With Respect to Fluoride Contamination in Industrial Area of Angul District Orissa India. Indian Journal of Scientific Research and Technology, 1, 54-61. http://www.indjsrt.com/administrator/modules/category/upload/10-15.pdf

[8] Kotoky, P., Barooah, P.K., Baruah, M.K., Goswami, A., Borah, G.C., Gogoi, H.M., Ahmed, F., Gogoi, A. and Paul, A.B. (2008) Fluoride and Endemic Fluorosis in the Karbianglong District, Assam, India. Fluoride, 41, 42-45. http://www.fluorideresearch.org/411/files/FJ2008_v41_n1_p072-075.pdf

[9] Pathak, R.P., Pankaj, S., Sameer, V., Mahure, N.V., Rajeev, K. and Ratnam, M. (2012) Detection of Fluoride Contamination in the Surface and Sub-Surface Water near Thermal Power Station. International Journal of Engineering and Science, 1, 44-47. http://www.researchinventy.com/papers/v1i1/G011044047.pdf

[10] Guijian, L., Liugen, Z., Duzgoren-Aydin, N.S., Lianfen, G., Junhua, L. and Zicheng, P. (2007) Health Effects of Arsenic, Fluorine, and Selenium from Indoor Burning of Chinese Coal. Reviews of Environmental Contamination and Toxicology, 189, 89-106. http://dx.doi.org/10.1007/978-0-387-35368-5_4

[11] Ando, M., Tadano, M., Yamamoto, S., Tamura, K., Asanuma, S., Watanabe, T., Kondo, T., Sakurai, S., Ji, R., Liang, C., Chen, X., Hong, Z. and Cao, S. (2001) Health Effects of Fluoride Pollution caused by Coal Burning. Science of the Total Environment, 271, 107-116. http://dx.doi.org/10.1016/S0048-9697(00)00836-6

[12] Fidanci, U.R. and Sel, T. (2001) The Industrial Fluorosis Caused by a Coal-Burning Power Station and Its Effects on Sheep. Turkish Journal of Veterinary and Animal Science, 25, 735-741. http://journals.tubitak.gov.tr/veterinary/issues/vet-01-25-5/vet-25-5-16-0007-6.pdf

[13] Singaraja, C., Chidambaram, S., Anandhan, P., Prasanna, M.V., Thivya, C., Thilagavathi, R. and Sarathidasan, J. (2014) Geochemical Evaluation of Fluoride Contamination of Groundwater in the Thoothukudi District of Tamilnadu, India. Applied Water Science, 4, 241-250. http://dx.doi.org/10.1007/s13201-014-0157-y

[14] Singaraja, C., Chidambaram, S., Anandhan, P., Prasanna, M.V., Thivya, C. and Thilagavathi, R. (2013) A Study on the Status of Fluoride Ion in Groundwater of Coastal Hard Rock Aquifers of South India. Arabian Journal of Geosciences, 6, 4167-4177. http://dx.doi.org/10.1007/s12517-012-0675-6

[15] Pandey, A.C., Shekhar, S. and Nathawat, M.S. (2012) Evaluation of Fluoride Contamination in Groundwater Sources in Palamu District, Jharkhand, India. Journal of Applied Sciences, 12, 882-887. http://dx.doi.org/10.3923/jas.2012.882.887

[16] Kumar, A. and Kumar, V. (2015) Fluoride Contamination in Drinking Water and its Impact on Human Health of Kishanganj, Bihar, India. Research Journal of Chemical Sciences, 5, 76-84. http://www.isca.in/rjcs/Archives/v5/i2/13.ISCA-RJCS-2015-018.pdf

[17] Ghosh, S., Chakraborty, S., Roy, B., Banerjee, P. and Bagchi, A. (2010) Assessment of Health Risks Associated with Fluoride-Contaminated Groundwater in Birbhum District of West Bengal, India. Journal of Environmental Protection Science, 4, 13 - 21. http://aes.asia.edu.tw/Issues/JEPS2010/GhoshS2010.pdf

[18] Reddy, B.M., Sunitha, V. and Reddy, M.R. (2013) Fluoride and Nitrate Geochemistry of Groundwater from Kadiri, Mudigubba and Nallamada Mandals of Anantapur District, Andhra Pradesh, India. Journal of Agricultural Engineering and Biotechnology, 1, 37-42. http://www.academicpub.org/DownLoadPaper.aspx?PaperID=14051 http://dx.doi.org/10.18005/jaeb0102002

[19] Madhavan, N. and Subramanian, V. (2001) Fluoride Concentration in River Waters of South Asia. Current Science, 80, 1312-1319. http://www.iisc.ernet.in/currsci/may252001/1312.pdf

[20] Mamatha, S.V. and Haware, D.J. (2013) Document on Fluoride Accumulation in Ground and Surface Water of Mysore, Karnataka, India. Current World Environment, 8, 259-265. http://dx.doi.org/10.12944/CWE.8.2.11

[21] APHA, AWWA and WEF (2005) Standard Methods for the Examination of Water and Wastewater. 21st Edition, APHA, Washington DC, USA.

[22] BIS (2012) Drinking Water-Specification (ICS 13.060.20) 2nd Edition, Bureau of Indian Standard, New Delhi. http://cgwb.gov.in/Documents/WQ-standards.pdf

[23] WHO (2011) Guidelines for Drinking Water Quality. 4th Edition World Health Organization, Geneva, Switzerland. http://apps.who.int/iris/bitstream/10665/44584/1/9789241548151_eng.pdf 